\title{
MOLECULAR PERTURBATIONS
}

\author{
J. G. VALATIN \\ (PRÉSENTED BY P. GOMBAS: - RECEIVED 8 JUNE 1946.)
}

The interaction between rotation and electronic motion in a diatomic molecule is investigated by giving the direct wave mechanical form of the components of angular momenta. In order to get a suitable form of the interaction a reference system connected with the nuclei and independent from the electrons is used, defined by the Eulerian angles, in which the rectangular components of both total angular momentum and electronic angular momentum can be given. The adventage of this way of description in comparison with other used reference systems is emphasised. Observed perturbations are con. nected with a part of the effect of rotational coupling called here accidental, while there may be a considerable coupling between rotation and electronic motion without manifesting itself in irregularities. This is still more the case with respect to the coupling between vibration and electronic motion, since the nuclear motion may affect also the electronic part of the wave function with respect to which the averaging of the interactions of the particles leads to the potential curve characterising the regular sequence of energy levels. The fact that this electronic part of the wave function cannot be identified in general with an eigenfunction of the two-center problem, yields a term which can play essential rôle in homogeneous perturbations. The analogous and different features of homogeneous and heterogeneous perturbations are discussed.

The irregularities observable in the awaited sequence of molecular energy levels are the well-known perturbations in molecular spectra. Their observation is of great importance in the investigation of molecular term systems, yielding the possibility to the determination of characteristics of the perturbing electronic term system or to finding the connection between the positions of already known levels of different electronic states. The existence of the observed regular energy system of an electronic term of a diatomic molecule can be ascribed to the fact that owing to the much smaller mass of the electrons the motion of the nuclei can be considered to take place in a potential field averaged with respect to the electronic motions. Perturbations. observed between the levels of different electronic states can be then imputed to the coupling of electronic and nuclear motions owing to which this condition is no more fulfilled. Theoretical papers dealing with molecular perturbations determine the energy values and wave functions ascribed to the unperturbed energy states accordingly, neglecting in first approximation certain terms in the wave equation corresponding to this interaction, and interpret the observed perturbations as the effect of these neglected terms. In this way two kinds 
of effects can be interpreted showing a different character according to whether they are due to terms corresponding to the rotation or to the vibration of the nuclei.

In these interpretations of molecular perturbations some arbitrariness seems to be left in two directions. First, in answering the question, which part of the effect of the coupling between the different motions should be identified with the observed phenomenon of perturbations; secondly, in identifying the electronic part of the wave function, with respect to which the mentioned averagebility of the interactions between the particles leads to the potential curve of an electronic state, with the eigenfunctions of the two-center problem, dividing also in this way arbitrarily the coupling between nuclear vibration and electronic motion.

The situation is simpler with respect to the rotational coupling. Owing to the fact that the total angular momentum of the molecule is a constant of motion, the energy states of the molecule can be characterised by a given rotational quantum number $J$, and the effect of the coupling between rotation and electronic motion can be followed in the function of this quantum number $J$. This coupling leads for instance to the phenomena of the $A$-doubling and the rotational distortion of spin multiplets, and the size of its effect in comparison with other factors determines the intermediate position of the molecular state between Hund's different ideal coupling cases. But accordingly, there may be a strong coupling between rotation and electronic motion without becoming manifest in irregularities in the sequence of molecular levels, and though this coupling may give rise also to perturbations, its effect is already partly included in the positions of the unshifted terms.

The problem is still more complicated concerning the coupling of vibration and electronic motion. Here, owing to the dynamical character of molecular vibration, the vibrational quantum number $v$ has only an approximate significance which can be lost in the case of perturbations. Accordingly, the coupling effect cannot be followed in the function of any quantum number. On the other hand, also in the case when we have a regular vibrational structure and the nuclei can, therefore, be regarded as moving in a potential field given by an average of the interactions with respect to the electronic motion, since the motion of the nuclei is determined by an interaction between the electrons and the nuclei influenced by the motion of the electrons, it can be expected that also the electronic motion determining the averaged field will be influenced through the interaction by the motion of the nuclei. This is a point which seems to have been rather overlooked in supposing that the wave function factor describing the electronic motion can simply be identified with the wave 
function of the two-center problem resulting in the case of fixed nuclei. But as it can be shown, ${ }^{1}$ this is not the case in general, this supposition coming into contradiction with mathematical and experimental arguments. Consequently the interpretation of the corresponding perturbations is also to be essentially modified, as it should be shown in the second part of the paper.

The question of the coupling between rotation and electronic motion is a rather explained one. Kronig ${ }^{2,3}$, and Van Vleck ${ }^{4}$ gave a wave mechanical treatment of the question, while Born and Flügge ${ }^{5}$ pointed out referring to the theory of the rotation of a rigid top that the expression of the interaction can be dealt more simply by means of the angular momentum operators through matrix mechanics. Notwithstanding, perhaps it will not seem to be quite unuseful to investigate this question by welding the two methods, giving the wave mechanical form of the corresponiding angular momentum operators directly, but avoiding a reference to the properties of hypergeometric wave functions in determining the matrix elements. In this way it seems to be possible to get a clearer insight into the mechanism of the interaction by means of the expressions of the angular momenta and occasion offers to stress some points of not negligeable physical significance.

To investigate the diatomic molecule we introduce the coordinates of the molecular mass center and the relative coordinates of the particles related to the first atomic nucleus. The coordinates of the mass center which are of no interest from the point of view of the inner motion of the molecule can be separated, and neglecting correction terms resulting from the finite mass of the origin of the reference system the wave equation has the form

$$
\left\{T_{r}+T_{e}+U\right\} \Psi=E \Psi
$$

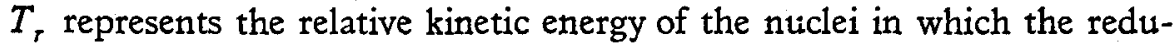
ced mass $\mu=\frac{M_{1} M_{2}}{M_{1}+M_{2}}$ of the nuclei enters, $T_{e}$ symbolises the kinetic energy of the electrons, while $U$ contains the interactions between all the particles, nuclei and electrons. In the description we use two kinds of coordinate systems. A system $x^{\prime}, y^{\prime}, z^{\prime}$ the axes of which are parallel with the axes of some inertia reference system, and a system $x, y, z$ the axes of which are given relative to the former by the Eulerian angles $\vartheta, \psi, \varphi$.

$1 \mathrm{~J}$. G. Valatin : Proc. Phys. Soc., 58, 695, 1946.

${ }^{2} R$. de L. Kronig: Z. f. Physik, 50, 347, 1928.

${ }^{3} R$. de L. Kronig : Band Spectra and Molecular Structure, 1930.

$4 \mathrm{~J}$. H. Van Vleck: Phys. Rev., 33, 467, 1929.

5 M. Born-S. Flügge : Ann. d. Physik (5), 16, 768, 1933. 
(Figure 1.) Identifying the $z$ axis of the second system with the line joining the two nuclei we have for the relative nuclear coordinates

$$
X_{r}^{\prime}=r \sin \vartheta \sin \psi \quad Y_{r}^{\prime}=-r \sin \vartheta \cos \psi \quad Z_{r}^{\prime}=r \cos \vartheta
$$

which would lead with $\psi^{\prime}=\psi-90^{\circ}$ to the usual description in polar coordinates. But in contrast to the description in Kronig's original paper and the paper of Born and Flügge, we do not identify the $x z$ plane with

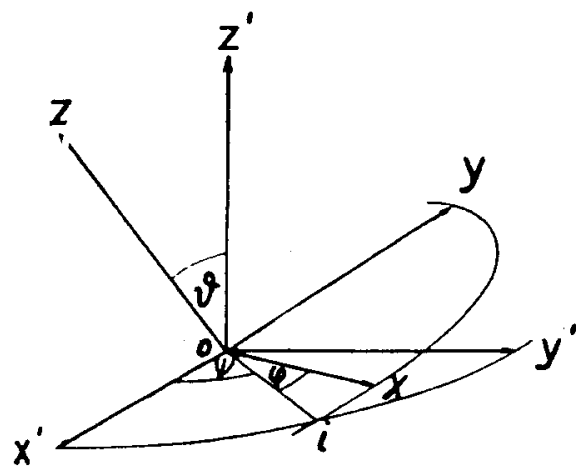

Fig 1 .

a plane containing a distinguished electron and preserve in this way the symmetry of the description in the electrons. As we shall see the choice of a suitable reference system plays important part in the due interpretability of the resulting expressions. As an introduction of electronic spin into the description does not give rise to any special difficulties we restrict ourselves for the sake of simplicity to singlet states and shall only indicate the differences caused by the spin. The finer effects connected with the direct dependence of the energy operator on electronic spin will be left completely out of consideration.

First let us take coordinates $r, \vartheta, \psi$ for the nuclei and $x_{i}^{\prime}, y_{i}^{\prime}, z_{i}^{\prime}$ 'cr the electrons in wave equation (1). Taking wave functions normalised with respect to $d r$ instead of $r^{2} d r$ the operator expression $\frac{1}{r^{2}} \frac{\partial}{\partial r}\left(r^{2} \frac{\partial}{\partial r}\right)$ in $T_{r}$ reduces to $\frac{\partial^{2}}{\partial r^{2}}$. Accordingly we have

$$
T_{r}=\frac{h^{2}}{8 \pi^{2} \mu}\left(-\frac{\partial^{2}}{\partial r^{2}}+\frac{1}{r^{2}} L^{(r) 2}\right)
$$

with

$$
L^{(r) 2}=-\left\{\frac{1}{\sin \vartheta} \frac{\partial^{\prime}}{\partial \vartheta}\left(\sin \vartheta \frac{\partial^{\prime}}{\partial \vartheta}\right)+\frac{1}{\sin ^{2} \vartheta} \frac{\partial^{\prime}:}{\partial \psi}\right\} \text {. }
$$


The prime on the differential operators indicates that the partial differentiation is to be carried out with fixed values of $x_{i}^{\prime}, y_{i}^{\prime}, z_{i}^{\prime}$. If $L_{x^{\prime}}^{(r)}, L_{y^{\prime}}^{(r)}$, $L_{z^{\prime}}^{(r)}$ are the operators for the components of nuclear angular momentum in $\frac{h}{2 \pi}$ units, we have

$$
L^{(r)^{2}}=L_{x^{\prime}}^{(r) 2}+L_{y^{\prime}}^{(r) 2}+L_{z^{\prime}}^{(r) 2}
$$

where with a simple transformation of the corresponding differential operators we can write

$$
\begin{aligned}
& L_{x^{\prime}}^{(r)}=-i\left\{Y_{r}^{\prime} \frac{\partial}{\partial Z_{r}^{\prime}}-Z_{r}^{\prime} \frac{\partial}{\partial Y_{r}^{\prime}}\right\}=i\left\{\cos \psi \frac{\partial^{\prime}}{\partial \vartheta^{\prime}}-\sin \psi \frac{\cos \vartheta}{\sin \vartheta} \frac{\partial^{\prime}}{\partial \psi}\right\} \\
& L_{y^{\prime}}^{(r)}=i\left\{Z_{r}^{\prime} \frac{\partial}{\partial X_{r}^{\prime}} \quad X_{r}^{\prime} \frac{\partial}{\partial Z_{r}^{\prime}}\right\}=i\left\{\sin \psi \frac{\partial^{\prime}}{\partial \gamma}+\cos \psi \frac{\cos \vartheta}{\sin \vartheta} \frac{\partial^{\prime}}{\partial \vartheta}\right\} \\
& L_{z^{\prime}}^{(r)}=i\left\{X_{r}^{\prime} \frac{\partial}{\partial Y_{r}^{\prime}} \quad Y_{r}^{\prime} \frac{\partial}{\partial X_{r}^{\prime}}\right\}=i \frac{\partial^{\prime}}{\partial \psi} .
\end{aligned}
$$

In order to bring the expression $U$ of the interaction energies to a simpler form containing no angular coordinates, let us now introduce the coordinates $x_{i}, y_{i}, z_{i}$. As it is seen by simple geometrical consideration the coordinate transformation takes place according to the following scheme:

\begin{tabular}{|c|c|c|c|}
\hline & $x^{\prime}$ & $y^{\prime}$ & $z^{\prime}$ \\
\hline$x$ & $\cos \varphi \cos \psi-\sin \varphi \sin \psi \cos \vartheta^{\prime}$ & $\cos \varphi \sin \psi+\sin \varphi \cos \psi \cos \vartheta$ & $\sin \varphi \sin \vartheta$ \\
\hline$y$ & $-\sin \varphi \cos \psi-\cos \varphi \sin \psi \operatorname{co} \psi$ & $-\sin \varphi \sin \psi+\cos \varphi \cos \psi \cos \vartheta$ & $\frac{\cos \varphi \sin \vartheta}{}$ \\
\hline$z$ & $\sin \psi \sin \vartheta$ & $-\cos \psi \sin \vartheta$ & $\cos \vartheta$ \\
\hline
\end{tabular}

The differential operators $\frac{\partial}{\partial x_{i}}, \frac{\partial}{\partial y_{i}}, \frac{\partial}{\partial z_{i}}$ are given by $\frac{\partial}{\partial x_{i}^{\prime}}, \frac{\partial}{\partial y_{i}^{\prime}}, \frac{\partial}{\partial z_{i}^{\prime}}$ according to the same scheme, and if we form the operators representing the components of the resultant electronic angular momentum along the $x^{\prime}, y^{\prime}, z^{\prime}$ respectively $x, y, z$ axes, we have

$$
\begin{array}{lr}
L_{x^{\prime}}=-i \Sigma\left(y_{i}^{\prime} \frac{\partial}{\partial z_{i}^{\prime}}-z_{i}^{\prime} \frac{\partial}{\partial y_{i}^{\prime}}\right) & L_{i}=-i \Sigma\left(y_{i} \frac{\partial}{\partial z_{i}}-z_{i} \frac{\partial}{\partial y_{i}}\right) \\
L_{y^{\prime}}=-i \Sigma\left(z_{i}^{\prime} \frac{\partial}{\partial x_{i}^{\prime}}-x_{i}^{\prime} \frac{\partial}{\partial z_{i}^{\prime}}\right) & \begin{array}{c}
\text { respec- } \\
\text { tively }
\end{array} L_{y}=-i \Sigma\left(z_{i} \frac{\partial}{\partial x_{i}}-x_{i} \frac{\partial}{\partial z_{i}}\right) \\
L_{z^{\prime}}=-i \Sigma\left(x_{i}^{\prime} \frac{\partial}{\partial y_{i}^{\prime}}-y_{i}^{\prime} \frac{\partial}{\partial x_{i}}\right) & L_{z}=-i \Sigma\left(x_{i} \frac{\partial}{\partial y_{i}}-y_{i} \frac{\partial}{\partial x_{i}}\right) .
\end{array}
$$


These are also transformed according to the scheme as sums of components of the vector product of covariantly transforming vectors of the three dimensional space. The well-known commutation rules are of course the same in the two systems.

$$
\begin{aligned}
& L_{x^{\prime}} L_{y^{\prime}}-L_{y^{\prime}} L_{x^{\prime}}=i L_{z^{\prime}} \\
& L_{y^{\prime}} L_{z^{\prime}}-L_{z^{\prime}} L_{y^{\prime}}=i L_{x^{\prime}} \\
& L_{z^{\prime}} L_{x^{\prime}}-L_{x^{\prime}} L_{z^{\prime}}=i L_{y^{\prime}}
\end{aligned}
$$

$$
\begin{aligned}
& L L_{y}-L_{y} L_{i}=i L_{z} \\
& L_{y} L_{z}-L_{z} L_{y}=i L_{x} \\
& L_{z} L-L L_{z}=i L_{y}
\end{aligned}
$$

Forming the expression of a total differential with the two kinds of variables and taking into account the relations between the variables, a comparison of the corresponding coefficients yields the relations

$$
\begin{aligned}
& \frac{\partial^{\prime}}{\partial \vartheta}=\frac{\partial}{\partial \vartheta}-i L_{o i}=\frac{\partial}{\partial \vartheta^{\prime}}-i L_{:} \cos \varphi+i L_{y} \sin \varphi \\
& \frac{\partial^{\prime}}{\partial \psi}=\frac{\partial}{\partial \psi}-i L_{z^{\prime}}=\frac{\partial}{\partial \psi}-i L_{:} \sin \varphi \sin \vartheta-i L_{y} \cos \varphi \sin \vartheta-i L_{z} \cos \vartheta \\
& \frac{\partial^{\prime}}{\partial \varphi}=\frac{\partial}{\partial \varphi}-i L_{z}
\end{aligned}
$$

where the operations $\frac{\partial}{\partial \vartheta}, \frac{\partial}{\partial \psi}, \frac{\partial}{\partial \varphi}$ are to be carried out by fixed $x_{i}, y_{i}, z_{i}$. If we would include also spin into the treatment, the spin coordinates could also be transformed to the new axes and would get the same result, $L_{x}, L_{y}, L_{z}$ representing in this case components of the resultant electronic angular momentum including spin. ${ }^{3}$ In view of this fact we shall denote in the following the quantum number corresponding to $L_{z}$ with $\Omega$, this meaning according to the accepted notation in the multiplet case the corresponding quantity including spin, while the corresponding quantum number without spin should be denoted by $A$.

Since in the original reference syscem the wave equation does not contain the variable $\varphi$, we have with respect to the molecular wave function $\Psi \boldsymbol{s}^{\prime}$,

$$
\frac{\partial^{\prime}}{\partial \varphi} \psi^{\prime}\left(x_{i}^{\prime}, r, \vartheta, \psi, \varphi\right) \equiv 0
$$

If, therefore, through the coordinate transformation $\psi^{\prime}$ is transformed into $\Psi, \Psi^{\prime}\left(x_{i}^{\prime}, r, \vartheta, \psi, \varphi\right) \longrightarrow \psi\left(x_{i}, r, \vartheta, \psi, \varphi\right)$, according to the last equation in (9) 


$$
-i \frac{\partial}{\partial \varphi} \Psi\left(x_{i}, r, \vartheta, \psi, \varphi\right)=L_{z} \Psi^{j}\left(x_{i}, r, \vartheta, \psi, \varphi\right) .
$$

Thus the introduction of the additional variable $\varphi$ involves the appearence of the relation (10) or (10a) which is invariant in time and selects those functions of the new variables which can represent a molecular state. For the whole functional space the operators $-i \frac{\partial}{\partial \varphi}$ and $L_{z}$ cannot be identified, as it can be seen also from the fact that while $L_{z}$ and $L_{x}$ obey the commutation rule (8b), $-i \frac{\partial}{\partial p}$ and $L_{x}$ commute.

Let us now write down the expressions for the components of the total angular momentum of the molecule along the axes of the inertia system. Using equations (5) and (9) and the transformation scheme (6) we get

$$
\begin{aligned}
& M_{x^{\prime}}=L_{x^{\prime}}^{(r)}+L_{x^{\prime}}=-i\left\{\cos \psi \frac{\partial}{\partial \vartheta}+\sin \psi \frac{1}{\sin \vartheta}\left(i L_{z}-\cos \vartheta \frac{\partial}{\partial \psi}\right)\right\} \\
& M_{y^{\prime}}=L_{y^{\prime}}^{(r)}+L_{y^{\prime}}=-i\left\{\sin \psi \frac{\partial}{\partial \vartheta}-\cos \psi \frac{1}{\sin \vartheta}\left(i L_{z}-\cos \vartheta \frac{\partial}{\partial \psi}\right)\right\} \\
& M_{z^{\prime}}=L_{z^{\prime}}^{(r)}+L_{z^{\prime}}=-i \frac{\partial}{\partial \psi} \\
& =-\left\{\frac{1}{\sin \vartheta} \frac{\partial}{\partial \vartheta}\left(\sin \vartheta \frac{\partial}{\partial \vartheta}\right)+\frac{1}{\sin ^{2} \vartheta}\left(\frac{\partial}{\partial \psi}-\cos \vartheta i L_{z}\right)^{2}-L_{z}^{2}\right\} .
\end{aligned}
$$

For the molecular states satisfying condition (10a) we can also write

$$
\begin{aligned}
& M_{x^{\prime}} \psi i=-i\left\{\cos \psi \frac{\partial}{\partial \vartheta}+\sin \psi \frac{1}{\sin \vartheta}\left(\frac{\partial}{\partial \varphi}-\cos \vartheta \frac{\partial}{\partial \psi}\right)\right\} \Psi s \\
& M_{y^{\prime}} \Psi j=-i\left\{\sin \psi \frac{\partial}{\partial \vartheta}-\cos \psi \frac{1}{\sin \vartheta}\left(\frac{\partial}{\partial \varphi}-\cos \vartheta \frac{\partial}{\partial \psi}\right)\right\} \Psi \\
& M_{z^{\prime}} \Psi=-i \frac{\partial}{\partial \psi} \Psi \\
& M^{2} \Psi=-i\left\{\frac{1}{\sin \vartheta} \frac{\partial}{\partial \vartheta}\left(\sin \vartheta \frac{\partial}{\partial \vartheta}\right)+\frac{1}{\sin ^{2} \vartheta}\left(\frac{\partial}{\partial \psi}-\cos \vartheta \frac{\partial}{\partial \varphi}\right)^{2}+\right. \\
& \left.\quad+\frac{\partial^{2}}{\partial \varphi^{2}}\right\}
\end{aligned}
$$


Since the total angular momentum is a constant of motion, the corresponding operators commute with the energy operator and we have simultaneously with the wave equation the equations

$$
\begin{aligned}
& M^{2} \psi=J(J+1) \Psi \xi \\
& M_{z^{\prime}} \psi=M \psi
\end{aligned}
$$

with the expressions (12a), (11a). It can be seen that according to the fact that equations (13) appear in connection with the invariance of the wave equation against a change of orientation of the coordinate system, they can be given in a form containing only the Eulerian angles $\vartheta, \psi, \varphi$ and the corresponding derivatives. This shows also the natural way for the separation of the angular variables.

Taking

$$
-i \frac{\partial}{\partial \varphi} \psi_{\Omega}=L_{z} \psi_{\Omega}=\Omega \psi_{\Omega}
$$

the first equation of (13) in its form given by (12a) multiplied by the factor $\frac{h^{2}}{8 \pi^{2} \mu} \frac{1}{r^{2}}$ gives the equation of Van Vleck's paper, occuring there as one of the equations resulting from an approximate separation of the wave equation and interpreted as the wave equation of the rigid symmetrical top. Here we can see its more general significance and its strict validity simu!taneously with the wave equation of the molecule.

According to the transformation (9) for $L^{(r) 2}$ we have from (

$$
\begin{gathered}
L^{(r) 2}=-\left\{\frac { 1 } { \operatorname { s i n } \vartheta } ( \frac { \partial } { \partial \psi } - i L _ { x } \operatorname { c o s } \varphi + i L _ { y } \operatorname { s i n } \varphi ) \operatorname { s i n } \vartheta \left(\frac{\partial}{\partial \psi}-\right.\right. \\
\left.-i L_{x} \cos \varphi+i L_{y} \sin \varphi\right)+\frac{1}{\sin ^{2} \vartheta}\left(\frac{\partial}{\partial \psi}-i L_{x} \sin \varphi \sin \vartheta-\right. \\
\left.\left.-i L_{y} \cos \varphi \sin \vartheta-i L_{z} \cos \vartheta\right)^{2}\right\}= \\
=-\frac{1}{\sin \vartheta} \frac{\partial}{\partial \vartheta}\left(\sin \vartheta \frac{\partial}{\partial \vartheta}\right)+\frac{1}{\sin ^{2} \vartheta}\left(\frac{\partial}{\partial \psi}-i L_{z} \cos \vartheta\right)^{2}+\left(L_{x}^{2}+L_{y}^{2}\right)+ \\
+2 i L_{x}\left[\cos \varphi \frac{\partial}{\partial \vartheta}+\sin \varphi \frac{1}{\sin \vartheta}\left(\frac{\partial}{\partial \psi}-i L_{z} \cos \vartheta\right)\right]+ \\
+2 i L_{y}\left[-\sin \varphi \frac{\partial}{\partial \vartheta}+\cos \varphi \frac{1}{\sin \vartheta}\left(\frac{\partial}{\partial \psi}-i L_{z} \cos \vartheta\right)\right]
\end{gathered}
$$


For molecular states satisfying (10a) we have with (12a)

$$
\begin{gathered}
L^{(r) 2} \psi j=\left\{M^{2}+L_{x}^{2}+L_{y}^{2}-L_{z}^{2}+2 i L\left[\cos \varphi \frac{\partial}{\partial \vartheta}+\right.\right. \\
\left.+\sin \varphi \frac{1}{\sin \vartheta}\left(\frac{\partial}{\partial \psi}-\cos \vartheta \frac{\partial}{\partial \varphi}\right)\right]+2 i L_{y}\left[-\sin \varphi \frac{\partial}{\partial \vartheta}+\right. \\
\left.\left.+\cos \varphi \frac{1}{\sin \vartheta}\left(\frac{\partial}{\partial \psi}-\cos \vartheta \frac{\partial}{\partial \varphi}\right)\right]\right\} \psi
\end{gathered}
$$

According to (11) we have in the primed system

$$
\begin{aligned}
L^{(r) 2}= & (M-L)^{2}=M^{2}+\left(L_{x^{\prime}}^{2}+L_{y^{\prime}}^{2}+L_{z^{\prime}}^{2}\right)- \\
& -2\left(L_{x^{\prime}} M_{x^{\prime}}+L_{y^{\prime}} M_{y^{\prime}}+L_{z^{\prime}} M_{z^{\prime}}\right) .
\end{aligned}
$$

Introducing the operators

$$
\begin{aligned}
& M_{\lambda}=-i\left\{\cos \varphi \frac{\partial}{\partial i}+\sin \varphi \frac{1}{\sin \vartheta}\left(\frac{\partial}{\partial \dot{\partial} \psi}-\cos \vartheta \frac{\partial}{\partial \varphi}\right)\right\} \\
& M_{y}=\cdots i\left\{-\sin \varphi \frac{\partial}{\partial \vartheta}+\cos \varphi \frac{1}{\sin \varphi}\left(\frac{\partial}{\partial \psi}-\cos \vartheta \frac{\partial}{\partial \varphi}\right)\right\} \\
& M_{z}=-i\left\{\frac{\partial}{\partial \psi}\right\}
\end{aligned}
$$

as it can be easily seen

$$
\left(M_{x}^{2}+M_{y}^{2}+M_{z}^{2}\right) \psi i=M
$$

is identical with the expression (12a), and accordingly $M_{x}, M_{y}, M_{z}$ can be interpreted as the rectangular components of the total angular momentum along the $x, y, z$ axes. Thus we have in analogy with (16)

$$
L^{(r) 2} \Psi j=\left\{M^{2}+\left(L_{x}^{2}+L_{y}^{2}-L_{z}^{2}\right)-2\left(L_{x} M_{x}+L_{y} M_{y}\right)\right\} \psi
$$

and in the expression (3) of the nuclear kinetic energy $T_{r}$, taking into account equation (13), the only term still containing the angular variables is represented by

$$
H_{L, M}=-\frac{1}{i \pi ! \mu} \frac{1}{r^{2}}\left(L_{x} M_{x}+L_{y} M_{y}\right) .
$$


Simple calculation gives the commutation rules

$$
\begin{array}{ll}
M_{x} M_{y}-M_{y} M_{x}=-i M_{z} & M_{x^{\prime}} M_{y^{\prime}}-M_{y^{\prime}} M_{x^{\prime}}=i M_{z^{\prime}} \\
M_{y} M_{z}-M_{z} M_{y}=-i M_{x} \text { (21a) } & M_{y^{\prime}} M_{z^{\prime}}-M_{z^{\prime}} M_{y^{\prime}}=i M_{x^{\prime}} \\
M_{z} M_{x}-M_{x} M_{z}=-i M_{y} & \text { while } \\
M_{z^{\prime}} M_{x^{\prime}}-M_{x^{\prime}} M_{z^{\prime}}=i M_{y^{\prime}}
\end{array}
$$

That is, the operators corresponding to the components of the total angular momentum along the rotating coordinate axes satisfy with an opposite sign the corresponding commutation rules along the axes of an inertia system. A result which can be found in the paper of O. Klein ${ }^{6}$ in the theory of a rigid asymmetric top. This relation between the commutation rules in the two systems can be also seen at a glance at the expressions (1 Ia) and (17) in which the complete analogy between the angular variables $\psi$ and $\varphi$ is disturbed only by a minus sign in the expression of $M_{y}$.

It should be noted that the rectangular components of angular momentum satisfying (18) have to be chosen according to (17), and here it is not allowed to replace $\frac{\partial}{\partial t p}$ by $i L_{z}$ given as a functional operator in $x_{i}$, $y_{i}, z_{i}$, since the term ctg $\vartheta \frac{\partial}{\partial y}$ occuring in the expression (12a) of $M^{2}$ appears in (18) just owing to the non-commutability of $\frac{\partial}{\partial \varphi}$ with $\sin \varphi, \cos \varphi$. By the same reason it can be seen that in a coordinate system, as used by Van Vleck, where the $x$ axis is identified with the intersection line oi of the planes $x^{\prime} y^{\prime}$ and $x y$ and thus the angle $\varphi$ does not occur, we get neither operators $M_{x}, M_{y}, M_{z}$ interpretable as rectangular components of the angular momentum, nor the corresponding simple form of the term (20). On the other hand, in using a reference system, as it is used in Kronig's original paper or by Born and Flügge, in which the $x z$ plane is identified with a plane containing the first electron, we disturb the symmetry in the electrons, and do not find operators $L_{x}, L_{y}, L_{z}$ which satisfy a simple commutation rule or could be interpreted as rectangular components of electronic angular momenta. ${ }^{7}$

O O. Klein: Z. f. Physik, 58, 730, 1929.

7 The corresponding terms in $L(r)^{2}$ can be partly seen in the quoted paper of Kronig in the expression $\delta_{\xi \eta \vartheta \vartheta \psi}=A_{\xi \eta \zeta} C_{\vartheta \varphi \psi}+B_{\xi_{\eta} \xi_{\varphi}} D_{\vartheta \varphi \psi}$ given under (2) and (3). Commuting the order of $A_{\xi \eta_{\zeta}}$ and $C_{\vartheta_{\varphi} \psi}$ respectively $B_{\xi \eta \zeta \varphi}$ and $D_{\vartheta \varphi \psi} A_{\xi \eta \zeta}$ is replaced by an expression $A_{\xi \eta \zeta}^{\prime}$ in which the last $\frac{\zeta_{1}}{\xi_{1}}$ term does not occur. We can intro-

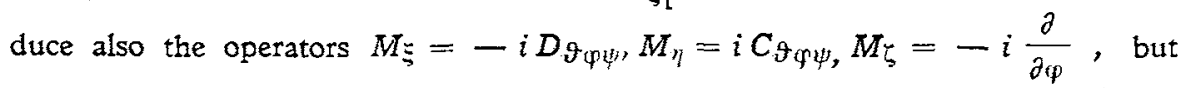


The adaptation of an appropriate reference system plays in this way an essential rô.e from the point of view of the simple interpretability of the energy terms (20) and this situation can be easily understood. Neglecting the terms under (20) a definite quantum number $\Omega$ can be given to every molecular state according to equation (14). The term (20) represents a coupling between rotation and electronic motion connecting states with different $\Omega$. The old quantum theory described the molecule by a model consisting of a rigid rotating body and an inserted symmetrical top the rotation of which corresponds to the electronic motion. ${ }^{8}$ Supposing an elastic coupling between the electronic top and the rigid body representing the nuclei the interaction between the two rotations can be described in quantum mechanics just by the expression (20). This expression, being a part of the nuclear kinetic energy, it is in connection with the rotation of the nuclei and its effect is the coupling of the angular momentum of the electronic top with the total angular momentum, owing to which states with different values of the angular momentum component of the electronic top along the nuclear axis are connected. According to the commutation rules (21a) and (8b) we get the special gyroscopic effect well-known from the spectra. But in order to see this simple interpretation of the interaction we have to use a reference system fixed to the nuclei with respect to which the electronic top may change its position. If in the case of a diatomic molecule we treat the nuclei as mass points we have to introduce an additional variable $\varphi$ in order to characterise a reference system fixed to the nuclei.

In analogy to the use of relative coordinates in wave mechanics where the position of the reference particle can also be given only by a probability distribution, we can also choose reference axes the positions of which are given only in a wave mechanical sense. We can speak in this sense of the component of a vector quantity along the nuclear axis of a diatomic molecule, and we can take rectangular coordinate systems of which all the three axes are given in such a way. The introduction of a new reference system means a coordinate transformation, and Hermitian

$-\frac{1}{2} i B_{\xi \eta_{\zeta} \varphi}$ and $\frac{1}{2} i A_{\zeta \eta \zeta}^{\prime}$ cannot be interpreted as $L_{\xi}$ respectively $L_{\eta}$, since in the expression of $L(r)^{2}$ there appears besides $-\frac{1}{4}\left(B^{2} \xi \eta \zeta \varphi+A^{\prime 2} \xi \eta \zeta\right)$ still an additional term $\frac{\zeta_{1}}{\xi_{1}} A_{\xi \eta \zeta}^{\prime}$ all of which are included there in the term $A_{\xi \eta \zeta R \varphi}$. Neither do these expressions give simple commutation rules together with the operator $L_{\zeta}=-i \frac{\partial}{\partial \varphi}$.

${ }^{8}$ H. A. Kramers-W. Pauli : Z. f. Physik, 13, 351, 1923. 
functional operators have to be interpreted as measurable quantities also in the functional space of the new variables. But if we choose the three axes of a rectangular reference system in an arbitrary way, we do not find in general functional operators which could be interpreted as the measurable quantities corresponding to the three rectangular components of a vector quantity along the axes of this reference system. Thus we can speak about the three rectangular components of the total angular momentum of a diatomic molecule also in a reference system the axes of which are fixed to the particles, but we cannot give rectangular components in a system of which one axis is connected with the nuclei and another axis is defined by an arbitrarily chosen plane $x^{\prime} y^{\prime}$. On the other hand, the three components of the resultant electronic angular momentum can be interpreted in systems of which the axes are given independently from the electrons, but operators corresponding to three rectangular components cannot be found if we connect one of the axes with the electrons in an arbitrary way. To a due description of the interaction between the total and electronic angular momenta, we have to choose a reference system satisfying both of these conditions.

In the chosen coordinate system in order to get wave functions $\psi \psi$ satisfying condition (10a) we take $\psi \psi$ as linear combination of wave functions $\Psi_{\Omega}$ satisfying equation (14). Neglecting the interaction (20) between rotation and electronic motion, these can be factorised according to

$$
\psi_{\Omega}\left(x_{i}, r, \vartheta, \psi, \gamma\right)=\Phi_{\Omega}\left(x_{i}, r\right) \Theta_{\Omega}^{J,}{ }^{M}(\vartheta, \psi, \varphi)
$$

where

$$
\Theta_{\Omega}^{J, M}(i,, \psi, \varphi)=\epsilon^{i M \psi} \epsilon^{i \Omega \psi} Q_{\Omega}^{J,}{ }^{M}(\cos \psi)
$$

is a solution of equations (13), (11a), (12a) and $Q_{S 2}^{J, M}$ is a hypergeometric polynom. For the determination of $\Phi_{\Omega}\left(x_{i}, r\right)$ we get from wave equation (1) the equation

$$
\begin{gathered}
\left\{\frac{h^{2}}{8 \pi^{2} \mu}\left(-\frac{\partial^{2}}{\partial r^{2}}+\frac{L_{x}^{2}+L_{y}^{2}-L_{z}^{2}}{r^{2}}+\frac{J(J+1)}{r^{2}}\right)+. T_{e}+U\right\} \Phi_{\Omega}\left(x_{i}, r\right)= \\
=E \Phi_{\Omega}\left(x_{i}, r\right) .
\end{gathered}
$$

In order to investigate the effect of the coupling term (20) let us introduce the operators

$$
\begin{array}{ll}
M_{p}=M_{x}+i M_{y} & M_{p^{\prime}}=M_{x^{\prime}}+i M_{y^{\prime}} \\
M_{q}=M_{x}-i M_{y} & M_{q^{\prime}}=M_{x^{\prime}}-i M_{y^{\prime}}
\end{array}
$$


The commutation rules (2la), (2lb) can be easily rewritten into

$M_{p} M_{q}-M_{q} M_{p}=-2 M_{z}$

$M_{q} M_{z}-M_{z} M_{q}=M_{p} \quad$ resp

$M_{p^{\prime}} M_{q^{\prime}} \quad M_{q^{\prime}} M_{p^{\prime}}=2 M_{z}$

$M_{q^{\prime}} M_{z^{\prime}} \quad M_{z^{\prime}} M_{q^{\prime}}=-M_{p^{\prime}}$

$M_{z} M_{p} \quad \cdots M_{p} M_{z}=M_{q} \quad$ tively $\quad M_{z^{\prime}} M_{p^{\prime}} \quad M_{p^{\prime}} M_{z^{\prime}}=-M_{q^{\prime}}$ 。

In this form it can be seen immediately that the consequence of the commutation rules of opposite sign is merely an interchange of the rôle of the operators $M_{p}$ and $M_{q}$. Thus with a correspondence $M_{q} \sim M_{p^{\prime}}, M_{p} \sim M_{q^{\prime}}$, $M_{z} \sim M_{z^{\prime}}$ we have in the system $x, y, z$ the same commutation rules as in $x^{\prime}, y^{\prime}, z^{\prime}$ and have the same well-known representations which give the matrix elements of the angular momentum expressions without the use of the hypergeometric wave functions. Accordingly, by the wellknown representations ${ }^{9}$ the non-vanishing matrix elements of $M_{p}, M_{q}$ are

$$
\begin{aligned}
& M_{p}(J, \Omega ; J \Omega-1)=\sqrt{J(J+1)-\Omega(\Omega \cdots 1)} \\
& M_{q}(J, \Omega ; J, \Omega+1)=\sqrt{J(J+1)-\Omega(\Omega+1)} .
\end{aligned}
$$

In wave mechanical form we have by (17)

$$
\begin{aligned}
& M_{p}=-i e^{-i \varphi}\left[\frac{\partial}{\partial i}+i \frac{1}{\sin i \phi}\left(\frac{\partial}{\partial \psi} \cdots \cos i \frac{\partial}{\partial \varphi}\right)\right] \\
& M_{q}=-i e^{i \varphi}\left[\frac{\partial}{\partial \vartheta}-i \frac{1}{\sin \psi}\left(\frac{\partial}{\partial \psi}-\cos i \frac{\partial}{\partial \varphi}\right)\right]
\end{aligned}
$$

which also show immediately by (22a), that only $M_{p}(J, \Omega ; J, \Omega-1)$ and $M_{q}(J, \Omega ; J, \Omega+1)$ can be non-vanishing matrix elements. The commutation rules given naturally involve already the commutation of $M_{p}, M_{q}$ with $M^{2}$.

Taking $L_{p}=L_{x}+i L_{y}, L_{q}=L_{x}-i L_{y}$, the interaction term can be written in the form

$$
H_{L, M}=-\frac{1}{8 \pi^{2} \mu} \frac{1}{r^{2}}\left(L_{p} M_{q}+L_{q} M_{p}\right) .
$$

It can be seen that $H_{L, M}$ has non-vanishing matrix elements only between states with the same $J$ and $M$ and with $\Omega^{\prime}=\Omega \pm 1$, which are yielded either by the first or by the second. term.

${ }^{9}$ For instance, B. L, van der Waerden : Die Gruppentheoretische Methode in der Quantenmechanik, 1932, S. 66. 


$$
\begin{gathered}
H_{L, M}(J, \Omega, u ; J, \Omega-1, v)= \\
=-\frac{1}{8 \pi^{2} \mu} \sqrt{J(J+1)-\Omega(\Omega-1)} \int \bar{\Phi}_{\Omega}^{(u)}\left(x_{i}, r\right) \frac{1}{r^{2}} L_{q} \Phi_{\Omega-1}^{(v)}\left(x_{i}, r\right) d x_{i} d r \\
=-\frac{1}{8 \pi^{2} \mu} \sqrt{J(J+1)-\Omega(\Omega+1)} \int \bar{\Phi}_{\Omega}^{(u)}\left(x_{i}, r\right) \frac{1}{r^{2}} L_{p} \Phi_{\Omega+1}^{(v)}\left(x_{i}, r\right) d x_{i} d r
\end{gathered}
$$

This shows also the variation of the interaction matrix elements with increasing rotational quantum number $J$ since this is defined first of all by the first factor.

The effect of this interaction between rotation and electronic motion can be of a considerable size without causing irregularities in the observed sequence of spectral terms, and the measure of the coupling may change between wide limits also with respect to the levels of the same electronic state. This means a regular mixing of the wave functions $\Psi_{\Omega}$ with different $\Omega$, owing to which $\Omega$ and $A$ hold more or less their quantum number rôle. In sfeaking about the interaction of states with definite $\Omega$ it is tacitly understood that if $|\Omega|>0$ the degeneracy causing $A$ type doublet is to be taken into account and the corresponding linear combinations of $\psi_{\Omega}$ and $\Psi_{-\Omega}$ form the starting point. As to the observed perturbations caused by the rotational coupling, these show a more accidental character, than the mentioned regular effect. This is a kind of resonance phenomenon, which presents itself if as a result of the different dynamic factors, two energy levels come into such a closeness of each other that the interaction (20) can get to a dominant rôle. Accordingly, the effect of the coupling between rotation and electronic motion can be considered as divided into two parts, a regular effect involving a regular displacement of the energy levels and being partly indistinguishable from the effect of the other parts of the energy operator, and an accidental effect observed as perturbations connected with the singular character of every perturbation process in the neighbourhood of coinciding approximate values. The irregular displacements of perturbed energy levels are connected with this second part of the effect of rotational coupling. As seen from the matrix elements (20b) the effect increases with increasing rotational quantum number and in the case of $J \rightarrow 0$ such rotational perturbations cannot be observed.

As to be seen, with sufficiently large $J$ owing to the mixing of the wave functions with different $\Omega$ we cannot separate the rotational part of the wave function, and we have to take into account the effect of coupling between rotation and electronic motion also in the case when this is not 
manifest in irregularities in the sequence of energy levels. The connection is still closer between nuclear vibration and electronic motion. Besides, while in the case of the rotational coupling, owing to the rigorous character of the angular momentum as a constant of motion, we can still follow the strengthening or loosening of this coupling with varying rotational quantum number, and can neglect the effect for $J \rightarrow 0$, in the case of the interaction of vibration and electronic motion we have a different situation. Vibration having purely dynamical character, the corresponding quantum numbers have only approximate significance which can also be completely loosed.

Starting now from equation (23) let us suppose $\Phi_{\Omega}\left(x_{i}, r\right)$ being factorisable according to

$$
\Phi_{\Omega}\left(x_{i}, r\right) \doteq f(r) \Phi_{e}^{(\Omega)}\left(x_{i}, r\right)
$$

with

$$
\int \bar{\Phi}_{\mathrm{e}}^{(\Omega)} \Phi_{e}^{(\Omega)} d x_{i} \equiv 1
$$

where the integration is to be meant through all the electronic coordinates. This normalisation means that in averaging with respect to the electronic motions the dependence on $r$ of the wave function is represented by the function factor $f(r)$. Averaging in this way equation (23) with respect to the motion of the electrons we get

$$
\left\{\frac{h^{2}}{8 \pi^{2} \mu}\left(-\frac{\partial^{2}}{\partial r^{2}}+\frac{J(J+1)}{r^{2}}\right)+V(r)-E\{f(r)=0\right.
$$

where $V(r)=V_{I}(r)+V_{I I}(r)$

$$
\begin{aligned}
& V_{I}(r)=\int \bar{\Phi}_{e}^{(\Omega)}\left(T_{e}+U\right) \Phi_{e}^{((2)} d x_{i} \\
& V_{I I}(r)=\frac{h^{2}}{8 \pi^{2} \mu} \int \bar{\Phi}_{e}^{(\Omega)}\left(-\frac{\partial^{2}}{\partial r^{2}}+\frac{L_{x}^{2}+L_{y}^{2}-L_{z}^{2}}{r^{2}}\right) \Phi_{e}^{(\Omega)} d x_{i} .
\end{aligned}
$$

Equation (28) is the wave equation of nuclear vibration in a potential field $V(r)$ yielded by an averaging of all the interactions between nuclei and electrons with respect to the electronic motions. This is in accordance with the experimental fact owing to which the levels of an electronic state show a regular system corresponding to a potential curve, and corresponds to the fact that owing to the much smaller mass of the electrons we can perform an approximate separation according to (27) and average the interactions of the particles with respect to the motion of the electrons: 
But as can be concluded by experimental and theoretical arguments, ${ }^{1}$ the functions $\Phi_{e}^{(\Omega)}$ cannot be identified in general with the eigenfunctions $\Phi_{0}^{(\Omega)}$ of the wave equation of the two-center problem, $\left\{T_{e}+U-E(r)\left\{\Phi_{o}^{(\Omega)}\left(x_{i}, r\right)=0\right.\right.$. The smaller mass of the electrons allows in general only an approximate separation according to (27), and does not justify the neglecting of the nuclear kinetic energy $T_{r}$ in wave equation (1), - which contains symmetrically the kinetic energies $T_{r}$ and $T_{e}$ besides the interaction $U$ of all the particles - in order to get to an approximation of $\Phi_{e}^{(\Omega)}$. The interaction energy $U$ represents the forces acting between nuclei and electrons, and not only the motion of the nuclei $i$; influenced according to (28), (29) by. the electronic motion described through the wave function $\Phi_{e}^{(\Omega)}$, but the nuclear motion can also have an influence on the motion of the electrons, that is on $\Phi_{e}^{(\Omega)}$, with respect to which we perform the averaging.

Writing the function (27) in equation (23), in passing to equation (28) we replaced with the corresponding averages the expressions

$$
\begin{aligned}
& \frac{h^{2}}{s \pi \mu}\left(-\frac{\partial^{2} f}{\partial r^{2}}+\frac{J(J+1)}{r^{2}} f\right) \phi_{e}^{(\ell)} \\
& \frac{h^{2}}{s \pi^{2} \mu} \frac{L_{x}^{\ddot{2}}+L_{y}^{\ddot{2}}-L_{z}^{2}}{r^{2}} f \Phi_{e}^{(! 2)} \\
& \frac{\hbar^{2}}{8 \pi^{2} \mu}\left(\cdots 2 \frac{\partial f}{\partial r} \frac{\partial \varphi_{e}^{(\Omega)}}{\partial r} \cdots f \frac{\partial^{2} \phi_{e}^{(\Omega)}}{\partial r^{2}}\right) \\
& \left(T_{e}+U\right) f \Phi_{e}^{(g)} .
\end{aligned}
$$

According to the approximate character of the factorisation (27) we have to try to obtain further approximations of wave equation (23) in taking into account the effect of these neglected term:

Suppesing with respect to different wave functions $\Phi_{e}^{(-2),(A)}$, $\Phi_{e}^{(\Omega)},(B), \int \Phi_{e}^{(D),(A)} \Phi_{e}^{(\Omega),(B)} d x_{i} \equiv 0$, the terms under $(30 \mathrm{a})$ do not give further contribution. This would be only the case, if $\Phi_{e}^{(\Omega),(A)}$ and $\Phi^{(\Omega),(B)}$ could not be considered orthogonal, which case cannot be a priori excluded. The term $(30 \mathrm{~b})$ represents still a part of the rotational coupling, connecting states with equal $\Omega$, and being the only term in (30) which has a part connecting also states with different $A$. Apart from this part of (30b) the terms in (30) couple states with equal $\Omega$ and equal $A$. The terms in (30c) containing derivatives of $\Phi_{e}^{(\Omega)}$ with respect to $r$, it is 
to be seen that their replacing with their average is allowed also in first approximation only in the case if the function $\Phi_{e}^{(\Omega)}$ does not suffer too rapid variations in $r$. This would be the case if we would identify $\Phi_{e}^{(\Omega)}$ with $\Phi_{0}^{(\Omega)}$ in the case of approximately crossing energy curves of the two-center problem, ${ }^{1}$ so that just in such cases essential differences are to be expected between $\Phi_{e}^{(\Omega)}$ and $\Phi_{a}^{(\Omega)}$, respectively between $V_{I}(r)$ and $E(r)$. With respect to the relative size of the terms in (30b) and (30c), according to an estimate of Van Vleck ${ }^{10}$ the first term in (30c) dominates the others with an order of magnitude. But with a similar reasoning we can see the possibility that the effect of the terms (30d) highly exceed the effect of the terms in $(30 \mathrm{~b})$ and $(30 \mathrm{c})$ if we have a suitable overlapping of he radial wave functions in the matrix elements

$$
\begin{gathered}
\int \bar{f}_{v_{1}}^{(\mathrm{A})}(r) \bar{\Phi}_{e}^{(\Omega),(A)}\left(x_{i}, r\right)\left(T_{e}+U\right) f_{v_{2}}^{(B)}(r) \Phi_{e}^{(g),(B)}\left(x_{i}, r\right) d x_{i} d r= \\
=\int \bar{f}_{v_{1}}^{(A)}(r) f_{v_{2}}^{(B)}(r) \int \bar{\Phi}_{e}^{(\Omega),(A)}\left(T_{e}+U\right) \Phi_{e}^{(\Omega),(B)} d x_{i} d r .
\end{gathered}
$$

These matrix elements give the reason for such strong interactions as in some observed homogeneous perturbations where the effect is larger in the order of magnitude than the effect for considerable $J$ values in heterogeneous perturbations. The corresponding effect being a mixing of wave functions with the same $\Omega$ of the form (27) in which the vibrational and electronic part of the wave function are separated, we may speak about a coupling between vibration and electronic motion, but owing to the form and origin of expression (30d) we could call the corresponding perturbations also electronic perturbations. By the mentioned identification of $\Phi_{e}^{(\Omega)}$ with the eigenfunctions of the two-center problem the matrix elements (31), which seems to have an important part in homogeneous perturbations, do not occur, owing to the diagonality of $T_{e}+U$ with respect to $\Phi_{0}^{(\Omega)}$.

Homogeneous perturbations observed between electronic states with equal $\Omega$ and $A$, can be thus interpreted by the effect of the nondiagonal par $\mathrm{t}$ of the terms (30) but with the same restriction as in the case of rotational perturbations. That is, not the whole effect presents itself as perturbations, but perturbations are just the manifestation of the corresponding accidental part of the effect. The situation is here all the more complicated since the interaction causing perturbations is larger in order of magnitude, and thus a single energy state is coupled with several states of another electronic state, whereas in the rotational case in general only the effect between two levels is of a considerable size. A due experimental

10 J. H. Van Vleck: J. Chem. Phys., 4, 327, 1936. 
explanation of the situation can be ascribed to L. Gerõ and R. Schmid in connection with the investigation of the perturbations of the $\mathrm{AgH}$ molecule. ${ }^{11}$ As they pointed out, ${ }^{12}$ the term vibrational perturbations is misleading in the sense that it has been often supposed that, since the interaction matrix elements depend only slightly on the rotational quantum number $J$, nor do vanish for $J=0$, the perturbations can be interpreted as the displacement of the vibrational levels with $J=0$ together with the rotational levels built up on them. That this is not at all the case can be seen either from molecular spectra by a suitable rotational analysis or by theoretical arguments. The terms (30b), (30c), (30d) containing not the rotational quantum number, the interaction matrix elements depend really only slightly through the corresponding variation of the wave functions on $J$, but perturbations occur always by a definite value of the rotational quantum number, and the size of displacement is defined by the accidental position of the levels at the given $J$. Perturbations corresponding to $J=0$ are in this way not at all characteristic to the perturbations resulting by other $J$ values. Levels with $J=0$ may be displaced or not, and their behaviour is in no way especially distinguished. Homogeneous perturbations show the same rotational structure as heterogeneous ones, with the difference that owing to the stronger interaction there is a simultaneous effect between several levels and that the change of peturbations with varying $J$ is chiefly only a consequence of the changing actual position of the interacting levels. It can also be seen from the perturbations of $A g H$ that the vibrational quantum number $V$ can loose its significance completely, the actual wave functions being linear combinations of several wave functions with different $v$. Since before the suitable analysis of the $A g H$ spectrum there were many unsettled questions concerning the character of homogeneous perturbations, the corresponding litterature should be accordingly revised. Thus for instance the corresponding pages of Herzberg's excellent book should be completely rewritten. ${ }^{13}$

Dieke distinguishes two classes of perturbations. ${ }^{14}$ Class $A$ perturbations corresponding to the rotational coupling, and class $B$ perturbations interpreted in this paper by the terms (30). Accepting this distinction we can in summarising state that both effects are characterised first of all by factors which we have called here accidental. These vary with varying $J$, the perturbations occuring always between states of definite $J$. In the case of class $A$ perturbations also the corresponding matrix elements are func-

11 L. Gerô-R. Schmid: Z. f. Phys., 121, 459, 1943. 835,1942 .

12 L. Gerõ-R. Schmid : Mathematikai és Természettudományi Értesítő, LXI.,

${ }_{13}$ G. Herzberg : Molecular Spectra and Molecular Structure, 1939, pp. 319-326.

14 G. H. Dieke: Phys. Rev., 47, 870, 1935. 
tions of $J$, and the effect vanishes for $J \rightarrow 0$. In the case of class $B$ perturbations the matrix elements depend only little on $J$, and the character of perturbations may be a quite different one owing to an interaction which differs in order of magnitude. Class $A$ perturbaticns are also called heterogeneous occuring between states with different $\Omega$, class $B$ perturbations are also called homogeneous resulting between states with equal $\Omega$. Dieke's distinction as a phenomenological one is more suitable in the sense, that neither $\Omega$ nor $A$ having a rigorous meaning in general, perturbations may be caused by both effects, the character of perturbations being defined by the more dominating factor.

Institute of Experimental Physics, Hungarian University for Technical and Economic Sciences, Budapest. 